\title{
Combined Influence of Noncondensable Gas Mass Fraction and Mathematical Model on Cavitation Performance of Bearing
}

\author{
Lili Wang $\mathbb{D}$, Zengkai Liu, Guoteng Yuan, and Yuliang Wei \\ College of Mechanical and Electronic Engineering, Shandong University of Science and Technology, Qingdao 266590, China \\ Correspondence should be addressed to Lili Wang; wang-ly-ly@163.com
}

Received 28 July 2020; Revised 27 September 2019; Accepted 26 October 2019; Published 23 January 2020

Academic Editor: M. Razi Nalim

Copyright (c) 2020 Lili Wang et al. This is an open access article distributed under the Creative Commons Attribution License, which permits unrestricted use, distribution, and reproduction in any medium, provided the original work is properly cited.

\begin{abstract}
The presence of cavitation in the oil film seriously affects the bearing lubrication performance and bearing capacity. Now the research of this phenomenon mostly focuses on the model of Reynolds equation (R-E equation) or Navier-Stokes equation (N-S), the influence of the two computation models is less analyzed, and the effect of noncondensable gas (NCG) mass fraction on the bearing performance is seldom studied. In the manuscript, the cavitation mechanism is studied using the mixed model of threedimensional N-S equation and Jakobsson-Floberg-Olsson (JFO) condition of two dimensional Reynolds equation, and the influence of rotational speed and NCG mass fraction on the cavitationoil film pressure, and bearing capacity was studied. The results show that the change trend of cavitation with the rotational speed is basically consistent for N-S equation and R-E equation. The bearing capacity calculated by N-S equation is greater than that calculated by R-E equation. The peak pressure and bearing capacity of film can be improved by increasing the NCG mass fraction of lubricant and rotational speed.
\end{abstract}

\section{Introduction}

The presence of cavitation in the oil film seriously affects the bearing lubrication performance and bearing capacity. The occurrence of cavitation causes noise, vibration and corrosion of bearing, which affects the stable operation and service life of the bearing. Therefore, scholars have carried out a series of studies on cavitation phenomena, such as experimental research, theoretical analysis, and numerical calculation.

In the experimental study, Brewe [1] used the high speed camera to study the dynamic cavitation characteristics of the bearing oil film. It pointed out that the smaller the bearing clearance, the higher the journal rotation speed and the greater the eccentricity, the more likely the cavitation. Fu et al. [2] used the mass conserving cavitation model to simulate the cavitation regions, the results of the model are validated by the experimental data from the literature. Morris et al. [3] showed that improved conditions with a textured journal, delaying the effect of lubricant rupture, thus extending the effective region of bearing load. Poddar et al. [4] got a clearer picture of the film by filming the moment of the film, using vibration and sound technology to monitor the formation of cavitation. Wang et al. [5] studied the shape, location, and characteristics of the spiral wedge journal bearing with transparent bearing and high speed camera, and pointed out that the higher speed and the lower oil supply pressure will lead to the obvious increase of oil film rupture area.

In the theoretical research, Dhande et al. [6] found that the maximum pressure value of the bearing decreased, when cavitation was considered. Ramos et al. [7] used finite element method to study the cavitation effect of hydrodynamic sliding bearing, the results showed that the addition of mass conservation in the cavitation process had little impact on the dynamic response of rotor under normal working conditions. Aggarwal et al. [8] studied frictional and load-carrying behaviours of micro-textured sector shape pad thrust bearing considering the cavitation and thermal effects. Cheng et al. [9] pointed out that the slip velocity of the cavitation zone would affect the oil film pressure, bearing capacity, and dynamic coefficient. Gao et al. [10] analyzed the relationship between eccentricity and sommerfeld number of water lubricated bearings using the cavitation model. Li et al. [11] proposed a new cavitation model based on air solubility, which provided a new idea for the cavitation model development of low vapor pressure lubricant. Guo et al. [12] established a three-dimensional gas-liquid mixed-phase CFD model, and analyzed the effect 


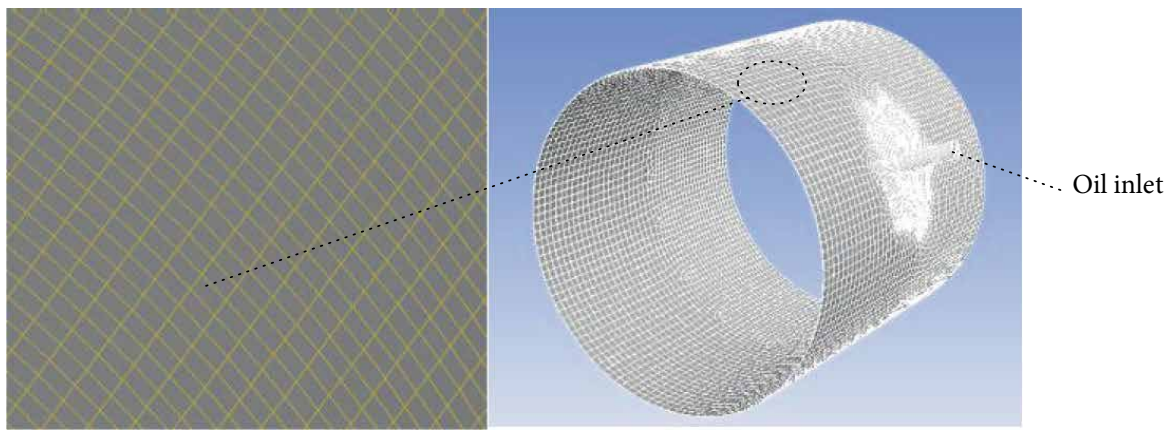

Figure 1: The structure of model and division of grid.

of noncondensing gas on the pressure field and phase field using a fully cavitated model. Christian et al. [13] studied the pressure, the axial track, and the friction loss of the axial and circumferential grooves journal bearing, based on the three-dimensional N-S equation. Ji and Cheng et al. [14, 15] studied numerically the cavitating flow around a NACA66 hydrofoil, and carried out numerical simulations of a tip leakage cavitating flow of a straight NACA009 hydrofoil.

In the actual work process, lubricating oil circulates in the oil supply system, strongly entrains and mixes with the air of tubing, which leads to the lubricant containing some air. Air is a noncondensable gas under normal conditions, and the effect of NCG mass fraction on the bearing performance is seldom studied. In the manuscript, the influence of different rational speeds and NCG mass fractions on the bearing performance is studied and compared. Scholars have studied the cavitation phenomenon using Reynolds equation or N-S model, the influence of the two computation models is less analyzed. So a better analysis for bearing cavitation performance is finished using the advantages of the three-dimensional N-S model and two-dimensional Reynolds equation.

\section{Numerical Model}

2.1. Establishment of N-S Equation. The basic equations used in the study of fluid lubrication are mainly Navier-Stokes equations, substantive expressions are as following [16]:

$$
\begin{gathered}
\frac{\partial \rho}{\partial t}+\frac{\partial}{\partial x_{i}}\left(\rho u_{i}\right)=0 \\
\frac{\partial}{\partial_{t}}\left(\rho u_{i}\right)+\frac{\partial}{\partial x_{j}}\left(\rho u_{i} u_{j}\right)=-\frac{\partial p}{\partial x_{i}}+\frac{\partial}{\partial x_{j}}\left(\mu \frac{\partial u_{i}}{\partial x_{j}}\right)+\rho F_{i},
\end{gathered}
$$

where $t$ is the time, $u$ is the fluid velocity, $F$ is the mass force per unit mass of fluid, $\rho$ is the density, $p$ is the fluid pressure. $i, j$ are $x, y, z$ three directions.

The finite element software is usually used to solve the N-S equation, Fluent software is used to analyze the bearing model, determine the calculation field, and Gambit is used to establish the model and grid. Since the thickness of the oil film is very small compared to the diameter and width, the thickness of the oil film has a great influence on the entire analysis, the model is divided into meshes by block partition. The grid gap

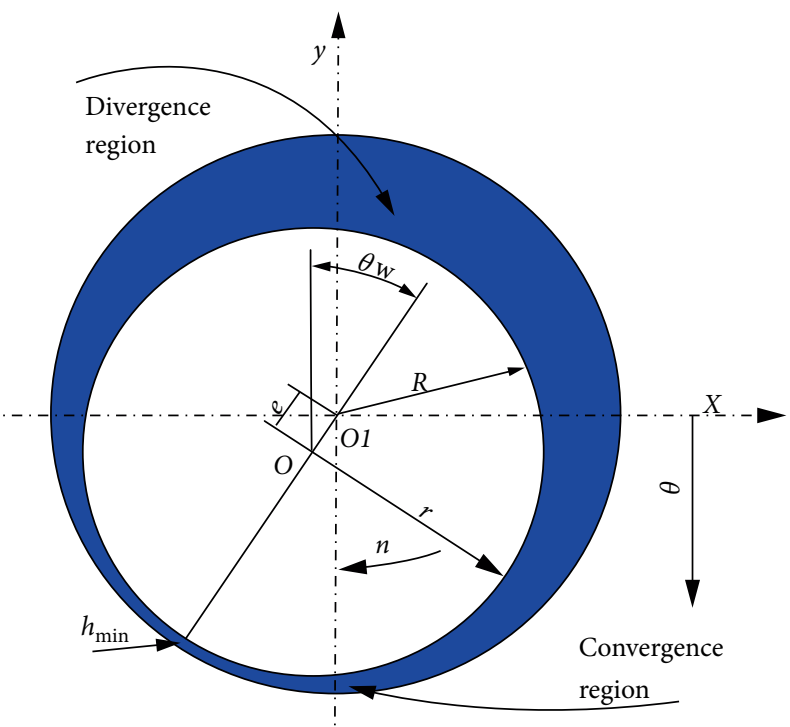

FIGURE 2: The geometrical structure of journal bearing.

is set to 0.5 , and 6 layers are arranged in the direction of oil film thickness. The oil film part is divided into a regular hexahedral mesh, the contact region of the inlet and an unstructured mixed grid, which guarantees a high grid quality. Model structure and division of grid are shown in Figure 1, the bearing geometry is shown in Figure 2.

2.2. Establishment of Two Dimensional Reynolds Equation. Assume that the lubricant is constant viscosity, laminar, neglecting the layer weight, and the inertial force during movement. The Reynolds equation is obtained by simplifying the N-S equation:

$$
\frac{\partial}{\partial x}\left(\frac{\rho h^{3}}{\mu} \frac{\partial p}{\partial x}\right)+\frac{\partial}{\partial z}\left(\frac{\rho h^{3}}{\mu} \frac{\partial p}{\partial z}\right)=6 U \frac{\partial(\rho h)}{\partial x}+12 \rho \frac{\partial h}{\partial t},
$$

where $\mu$ is lubricating oil viscosity, $p$ is oil film pressure, $U$ is rotating speed of bearing, $h$ is oil film thickness.

Common boundary conditions for the Reynolds equation have the whole Sommerfeld boundary condition, the semi-Sommerfeld boundary condition, the Reynolds boundary condition, and the mass conservation boundary condition. The Reynolds boundary condition is often used, it cannot 


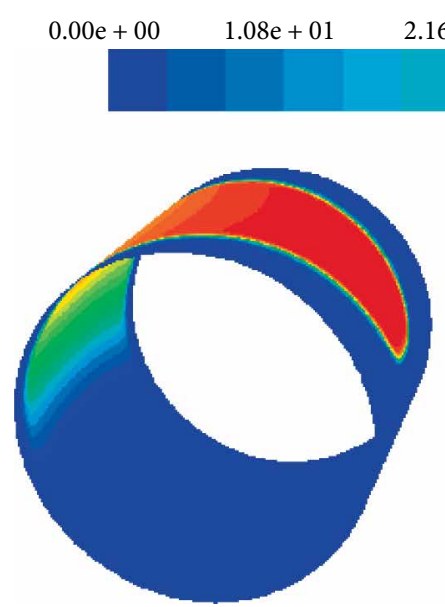

(a)

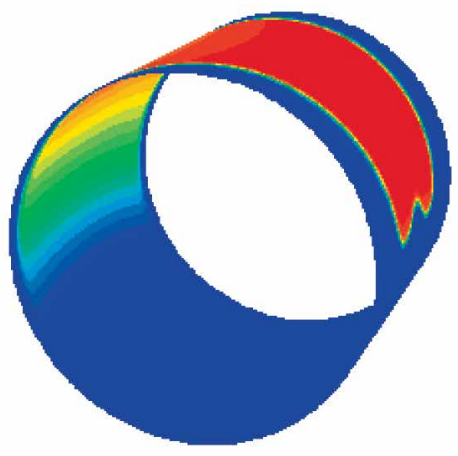

(b)

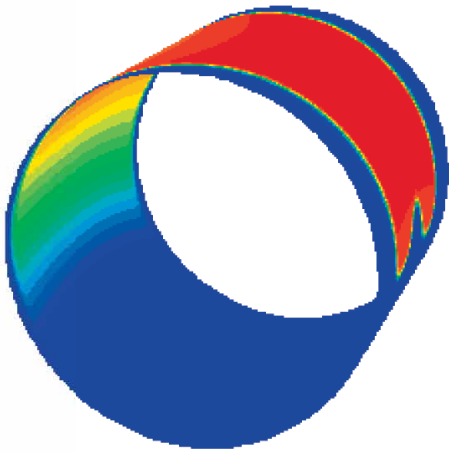

(c)

FIGURE 3: Influence of rotating speed $n$ on the volume fraction of cavitation in N-S model. (a) $n=2000 \mathrm{r} / \mathrm{min}$. (b) $n=4000 \mathrm{r} / \mathrm{min}$. (c) $n=6000 \mathrm{r} / \mathrm{min}$.

interpret the oil film reformation correctly and does not respect conservation of mass, and mass conserving boundary condition (JFO boundary condition) that is presented by Jakobsson, Floberg, Olsson considers mass conservation in oil film rupture and reformation location. In this manuscript, the JFO boundary condition with the cavitation pressure $\rho_{\text {cav }}$ of $-72139.79 \mathrm{~Pa}$ is used [17].

Elrod algorithm is used in Equation (3) for solving the JFO boundary condition. The switch function $g$ is used to revise the Reynolds equation and density as a dependent variable instead of pressure. Nondimensional density $\alpha_{\rho}=\rho / \rho_{\text {cav }}$ is defined. For using density as a dependent variable instead of pressure in Reynolds equation, the Elord algorithm treats lubricating oil as a compressible fluid with a large modulus of elasticity, introducing the nondimensional unit step function $g$ into the relationship equation of density and pressure, $\beta=\rho \partial \rho / \partial \rho$ :

$$
g \beta=\frac{\rho}{\rho_{\text {cav }}} \frac{\partial p}{\partial\left(\rho / \rho_{\text {cav }}\right)}=\alpha_{\rho} \frac{\partial p}{\partial \alpha_{\rho}}
$$

Substituting $x=R \phi, \lambda=z / L, \bar{h}=h / c, \bar{\beta}=\left(c^{2} / 6 U \eta R\right) \beta$ and Equation (4) into Equation (3), the nondimensional Reynolds equation considering cavitation effect is as following:

$$
\begin{aligned}
& \frac{\partial}{\partial \phi}\left(\bar{h}^{3} g \bar{\beta} \frac{\partial \alpha_{\rho}}{\partial \phi}\right)+\left(\frac{1}{2 X i}\right)^{2} \frac{\partial}{\partial \lambda}\left(\bar{h}^{3} g \bar{\beta} \frac{\partial \alpha_{\rho}}{\partial \lambda}\right) \\
& \quad=\frac{\partial\left(\alpha_{\rho} \bar{h}\right)}{\partial \phi}+2 \frac{\partial\left(\alpha_{\rho} \bar{h}\right)}{\partial t},
\end{aligned}
$$

where $\bar{\beta}$ is nondimensional elastic modulus of lubricant, $\bar{h}$ is nondimensional oil film thickness, $\lambda$ is nondimensional axial direction, $X i$ is width-diameter ratio, $L$ is bearing width, $\rho_{\text {cav }}$ is cavitation density, and $g$ is 0 in the cavitation region; $g$ is 1 in the full oil film region.

The two-dimensional Reynolds equation is solved by the finite difference method and the discretization Equation (5), the discretization equations are solved by the superrelaxation iterative method, the calculation process stopped using the criteria of pressure smoothness and the iterative accuracy of bearing capacity. After solving Equation (5) to obtain $\alpha_{\rho}$, the pressure distribution needs to be solved by Equation (4).

\section{Numeral Calculations}

For the three-dimensional model, Fluent provides three common cavitation models, Volume of Fluid (VOF model), Mixture, and Eulerian. Mixture is a simplified multiphase flow model that can be used to simulate two-phase or multiphase flow with different speeds (particles or fluids), which is suitable for load flow of low-mass load particle, bubble flow. In this manuscript, Mixture (mixed model) is used, fluid is treated as incompressible due to the change of fluid density it can be ignored, lubricant density $\rho=890 \mathrm{~kg} / \mathrm{m}^{2}$, the shaft rotates at rotating speed $n$ and the bearing is stationary. The boundary conditions are as follows: the inlet pressure is $20 \mathrm{kPa}$, the outlet is both sides of oil film and the pressure is environmental pressure. The finite volume method is used to discretize Equation (2), the residual convergence is set to $10^{-4}$ as iterative stop.

The model parameters are calculated as follows: bearing radius $R=20.05 \mathrm{~mm}$, journal radius $r=20 \mathrm{~mm}$, radius clearance $c=0.05$, eccentricity $\varepsilon=0.6$, inlet diameter $d=2 \mathrm{~mm}$, inlet pressure $P_{\text {in }}=20 \mathrm{kPa}$, rotating speed $n=2000 \mathrm{r} / \mathrm{min}, 4000 \mathrm{r} /$ $\mathrm{min}, 6000 \mathrm{r} / \mathrm{min}$, lubricant viscosity $\mu=0.02 \mathrm{~Pa} . \mathrm{s}$, and initial offset angle $\theta_{w}=458$.

\section{Results and Discussion}

4.1. Effect of Rotational Speed on Cavitation. Figures 3 and 4 is the distribution of volume fraction of cavitation at different rotational speeds based on the N-S equation and R-E equation, separately. As can be seen from Figure 3, as the rotational speed increases, the large volume fraction cavitation gradually 


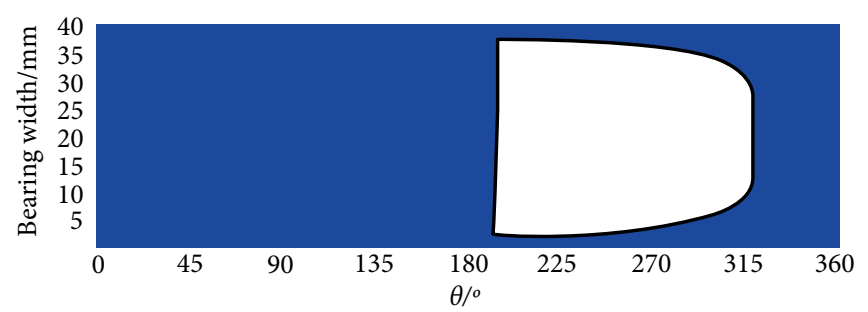

(a)

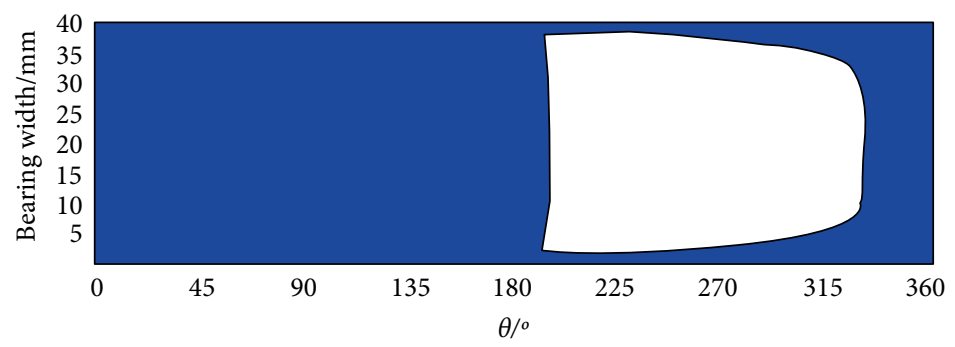

(b)

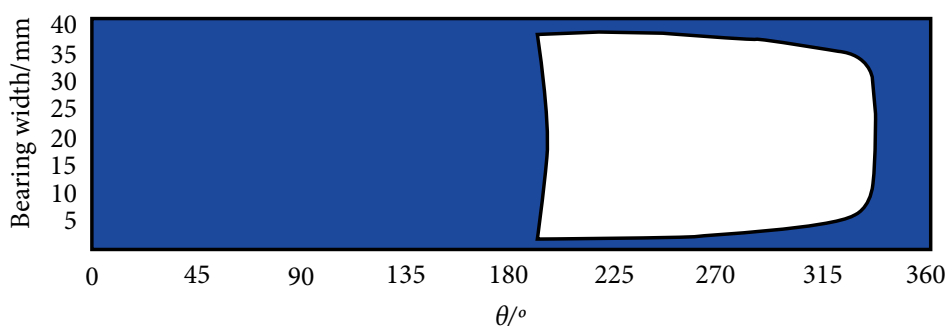

(c)

FIGURE 4: Influence of rotating speed $n$ on cavitation distribution in R-E model. (a) $n=2000 \mathrm{r} / \mathrm{min}$. (b) $n=4000 \mathrm{r} / \mathrm{min}$. (c) $n=6000 \mathrm{r} / \mathrm{min}$.

increases in the cavitation distribution cloud, and the cavitation phenomenon of oil film is more obvious. As it can be seen from Figure 4, the white region is cavitation, rotational speed will have a significant impact on the oil film characteristics, exacerbate the cavitation phenomenon of oil film, which is consistent with influence of rotating speed on cavitation in Figure 3. By computation for the ratio of cavitation area to oil film area in Figures 3, 4, and 5 shows the influence of the rotational speed on the cavitation area ratio and with the increase of rotational speed, the cavitation area ratio increases, which indicate that the number of cavitation constantly increases. Figure 5 is consistent with the changing trend of Figures 3 and 4, but the results based on the R-E equation are higher than those based on the N-S equation. The reason is as follows: R-E equation is the simplified form of N-S equation, $\mathrm{N}-\mathrm{S}$ equation can better distinguish the oil and gas mixing than $\mathrm{R}-\mathrm{E}$ equation in the cavitation computation, especially in the low volume fraction, R-E equation ignores the mixed lubricant and leads to higher calculation result.

It can be seen from Figure 6 that the trend of the N-S equation is consistent with the R-E equation as the journal rotation speed increases, the static pressure of the oil film has obviously increased, the bearing capacity has a corresponding increasing trend, but the angle of the oil film rupture is slightly different. Figure 6(a) is the oil film circumferential pressure distribution (starting point corresponds to the largest gap near the oil film), the oil film pressure is concentrated in the bearing

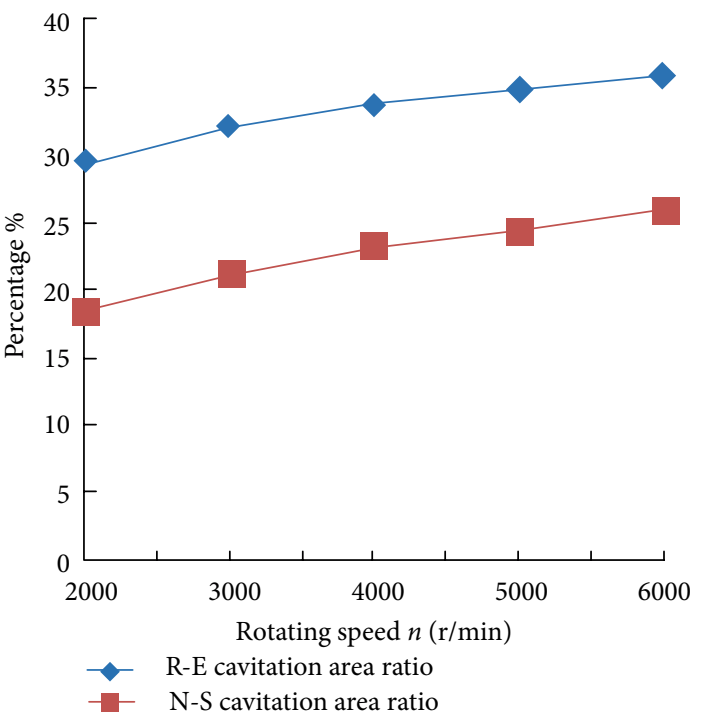

FIGURE 5: Influence of rotating speed on the area ratio of cavitation.

convergence area, oil film static pressure peak occurs before the minimum gap. When the negative pressure of the divergence zone reaches the saturated vapor pressure of the lubricating oil, cavitation is generated in the oil film, as shown in Figure 7. It can be seen from Figure 6(a) that the oil film pressure calculated using the R-E equation has a significant 


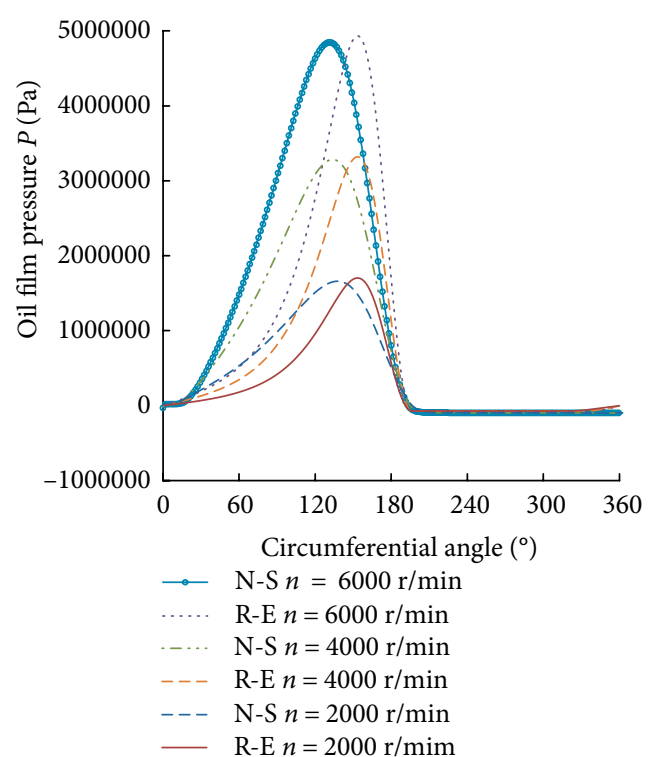

(a)

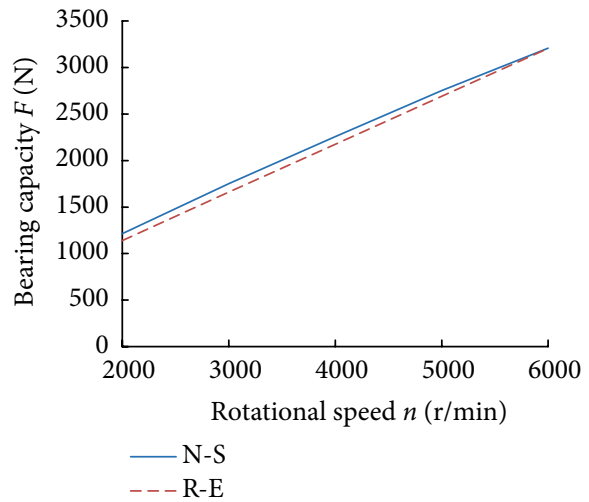

(b)

FIGURE 6: Effect of speed on oil film pressure and bearing capacity. (a) Oil film pressure. (b) Bearing capacity.
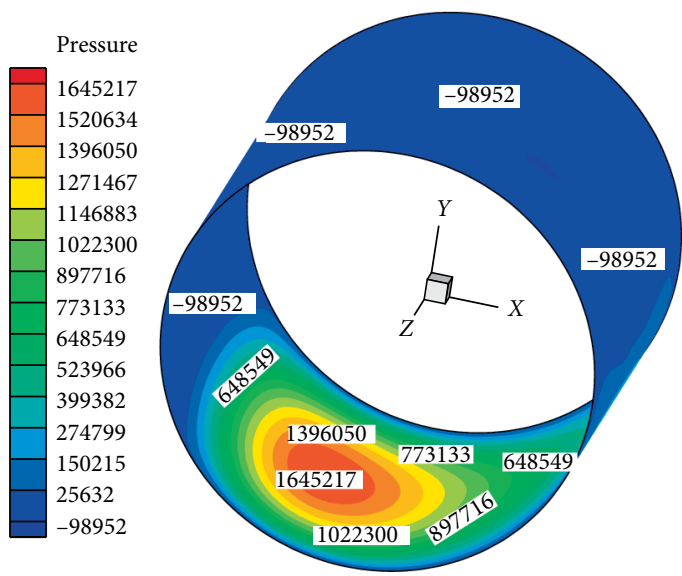

(a)

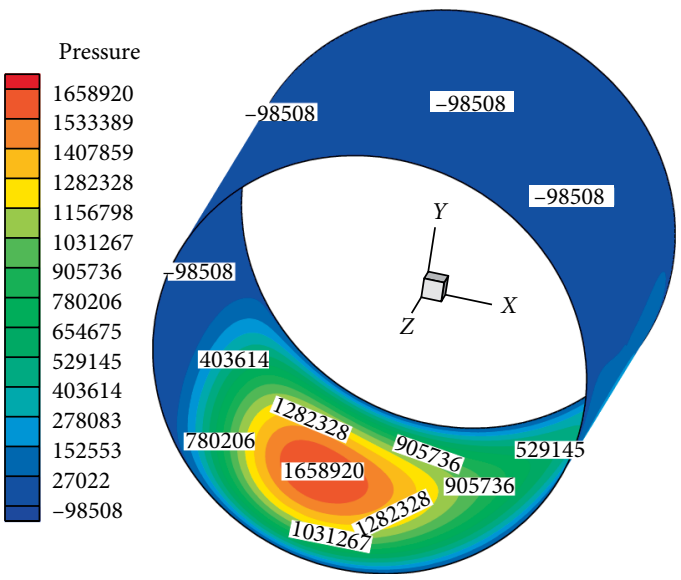

(c)
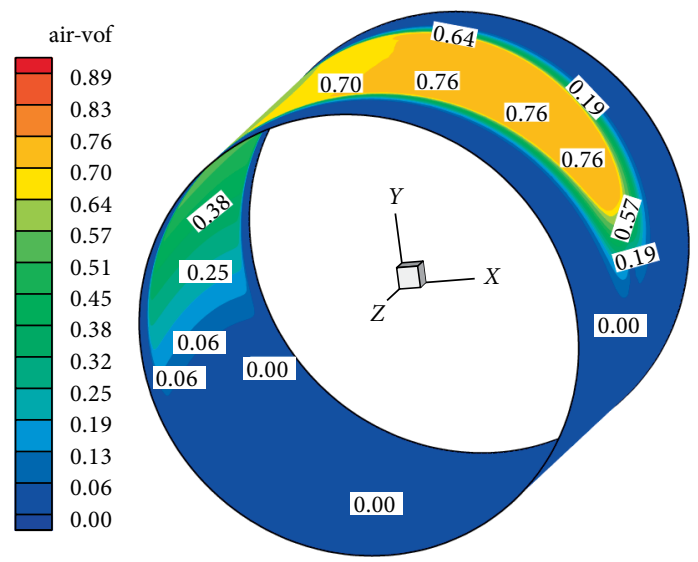

(b)
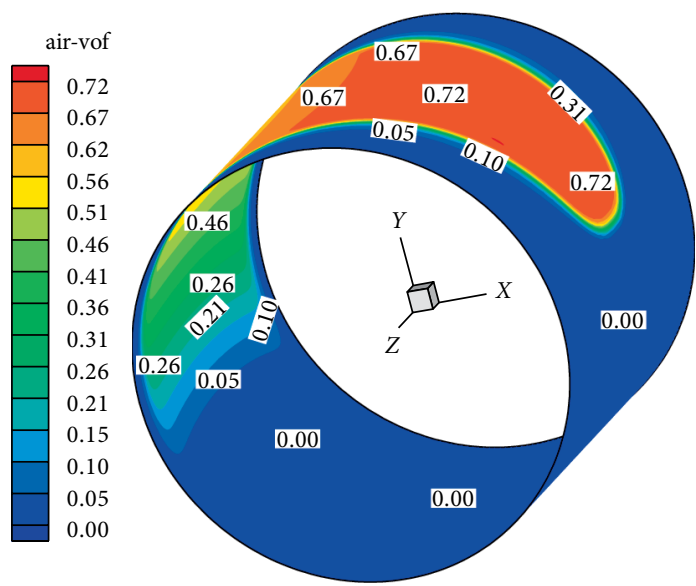

(d)

FIGURE 7: Effect of $w_{g}$ on oil film pressure and cavitation cloud $(n=2000 \mathrm{r} / \mathrm{min})$. (a) $w_{g}=1.5 \times 10^{-7}$ pressure distribution. (b) $w_{g}=1.5 \times 10^{-7}$ cavitation distribution. (c) $w_{g}=1.5 \times 10^{-5}$ pressure distribution. (d) $w_{g}=1.5 \times 10^{-5}$ cavitation distribution. 


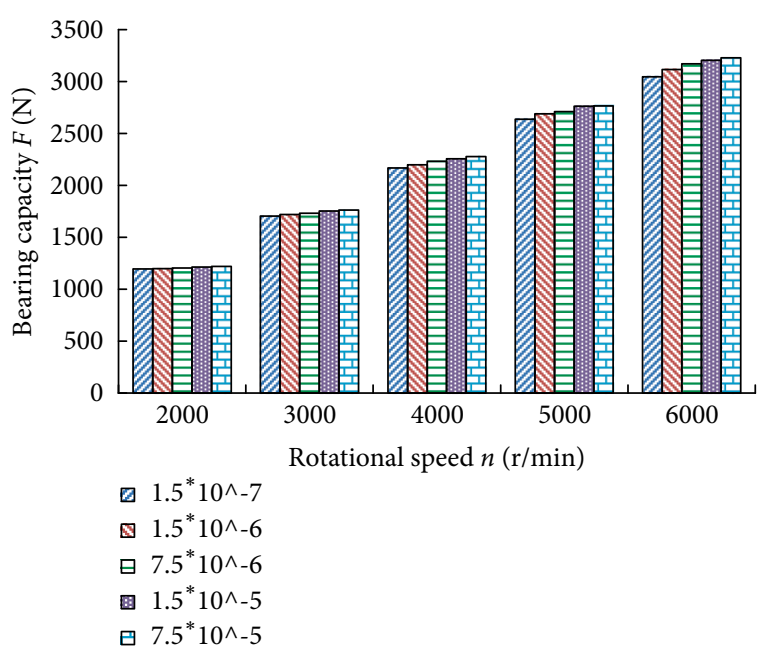

FIGURE 8: Effect of $w_{\mathrm{g}}$ and rotational speed on bearing capacity.

hysteresis, the peak value appears higher, the positive pressure area of the oil film decreases, and the oil film rupture area increases than that of the N-S equation. This is consistent with the cavitation ratio calculated in Figure 5 that the cavitation ratio of R-E equation is larger than the N-S equation. Since the oil film rupture region calculated by the R-E equation in Figure 6(a) is larger than the N-S equation, the bearing capacity calculated by the N-S equation shown in Figure 6(b) is greater than the bearing capacity of the R-E equation.

\subsection{Influence of NCG Mass Fraction on Bearing Perfor-} mance. The increase of axial rotational speed and the NCG mass fraction $W_{g}$ has certain influence on the peak value of static pressure and bearing capacity. From Figures 8 and 9, it can be seen that at the same NCG mass fraction, with the increase of rotation speed, the peak value of the static pressure and bearing capacity increase. Because of the increase of the rotational speed, more lubricating oil is brought into the wedge-shaped convergence gap, which enhances the hydrodynamic effect and carrying capacity of the bearing.

With the increase of the NCG mass fraction, the carrying capacity and the peak value of static pressure also increased slightly. It can also be seen from Figures 8 and 9 that the increase of NCG mass fraction has a greater increase range for the oil film pressure and bearing capacity at high rotational speed than at low speed. When the rotational speed is $2000 \mathrm{r} / \mathrm{min}$, the NCG mass fraction $w_{g}$ in the lubricating oil increases from $1.5 \times 10^{-7}$ to $7.5 \times 10^{-5^{g}}$, the pressure peak and the bearing capacity increase by $1.20 \%$ and $2.04 \%$, respectively. When the rotational speed is $6000 \mathrm{r} / \mathrm{min}$, the NCG mass fraction $w_{g}$ increases from $1.5 \times 10^{-7}$ to $7.5 \times 10^{-5}$, the pressure peak and carrying capacity of the oil film increase by $2.78 \%$ and $5.60 \%$, respectively. It is shown that the change of NCG mass fraction at high speed has greater impact on maximum static pressure of oil film and bearing capacity than at low speed.

As shown in Figures 7(a) and 7(c), with the increase of the NCG mass fraction in the lubricating oil, the maximum

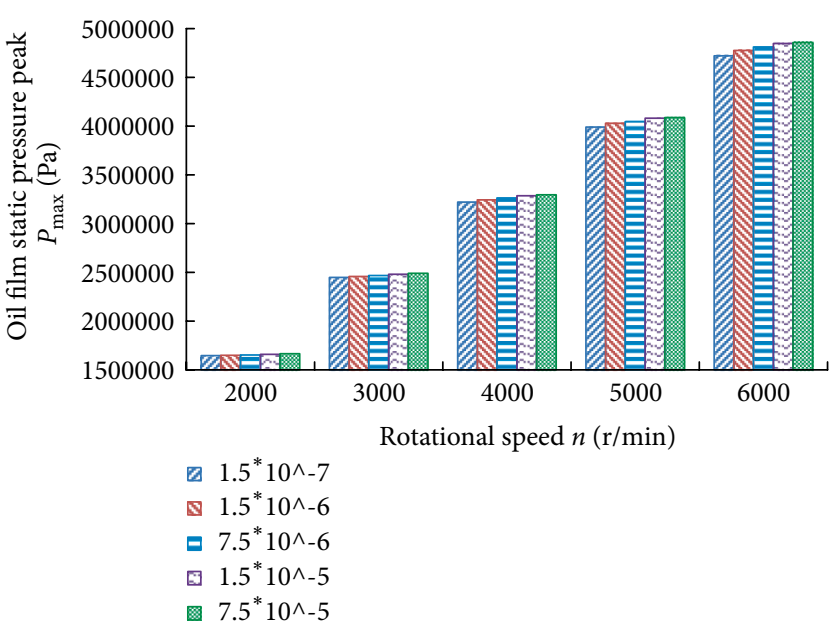

FIGURE 9: Effect of $w_{g}$ and rotational speed on oil film pressure peak.

positive pressure of the oil film in the convergent region gradually increases, and the maximum negative pressure in the divergent zone reduces. When the NCG mass fraction $w_{g}$ is $1.5 \times 10^{-7}$, the maximum positive pressure in the convergent region is $1645217 \mathrm{~Pa}$, and the maximum negative pressure in the divergent region is $-98952 \mathrm{~Pa}$. When the NCG mass fraction increases by 100 times and $w_{g}$ is $1.5 \times 10^{-5}$, the maximum static pressure of the oil film increases to $1658920 \mathrm{~Pa}$, the maximum negative pressure is $-98508 \mathrm{~Pa}$, and the variation of the maximum positive pressure and the maximum negative pressure are $0.83 \%$ and $0.45 \%$, respectively. It shows that the change of NCG mass fraction has little effect on the maximum positive pressure and maximum negative pressure of the oil film. It can be seen from (b) and (d) in Figure 7 that the maximum volume fraction of the cavitation area decreases with the increase of the NCG mass fraction, and the large volume fraction concentrates in the downstream position of the cavitation region. According to Figures 7(a) and 7(c), the increase of the NCG mass fraction reduces the maximum negative pressure in the oil film, restrains the further growth of cavitation, and then reduces the gas volume fraction.

\section{Conclusion}

Based on the mixed model of the three-dimensional N-S equation and JFO condition of two dimensional Reynolds equation, the influence of rotational speed, and NCG mass fraction on bearing cavitation performance is analyzed.

The calculated results of the N-S equation and the R-E equation on the cavitation are basically the same. With the increase of rotational speed, the oil film static pressure obviously increases, the bearing capacity also has a corresponding increase, the cavitation area gradually increases, and the cavitation area ratio also increases. The oil film pressure of the R-E equation has a significant hysteresis, and the pressure peak is higher than that calculated by the N-S equation. The cavitation area ratio of the R-E equation is higher than that calculated by the N-S equation, while the bearing capacity calculated by the N-S equation is greater than that calculated by the R-E equation. 
The increase of rotational speed has a significant effect on the oil film pressure and bearing capacity. The increase of noncondensable gas mass in the lubricating oil can increase the pressure peak value and bearing capacity.

\section{Data Availability}

The data used to support the findings of this study are available from the corresponding author upon request.

\section{Conflicts of Interest}

The authors declare that they have no conflicts of interest.

\section{Acknowledgments}

This work was supported by the grant from China Postdoctoral Science Foundation funded project (No. 2017M612304), Shandong Provincial Postdoctoral Innovation Foundation (No. 201701016), Qingdao Postdoctoral Research funded project, supported by SDUST Research Fund (No. 2015JQJH104).

\section{References}

[1] D. E. Brewe, "Theoretical modeling of the vapor cavitation in dynamically loaded journal bearings," Journal of Tribology, vol. 108, no. 4, pp. 628-637, 1986.

[2] G. Fu and A. Untaroiu, "An optimum design approach for textured thrust bearing with elliptical-shape dimples using computational fluid dynamics and design of experiments including cavitation," Journal of Engineering for Gas Turbines and Power, vol. 139, no. 9, p. 092502, 2017.

[3] N. J. Morris, H. Shahmohamadi, R. Rahmani, H. Rahnejat, and C. P. Garner, "Combined experimental and multiphase computational fluid dynamics analysis of surface textured journal bearings in mixed regime of lubrication," Lubrication Science, vol. 30, no. 4, pp. 161-173, 2018.

[4] S. Poddar and N. Tandon, "Detection of journal bearing vapour cavitation using vibration and acoustic emission techniques with the aid of oil film photography," Tribology International, vol. 103, pp. 95-101, 2016.

[5] L. L. Wang, Q. L. Zeng, C. H. Lu, and P. Liang, "A numerical analysis and experimental investigation of three oil grooves sleeve bearing performance," Industrial Lubrication \& Tribology, vol. 71, no. 2, pp. 181-187, 2019.

[6] D. Y. Dhande and D. W. Pande, "Multiphase flow analysis of hydrodynamic journal bearing using CFD coupled fluid structure interaction considering cavitation," Journal of King Saud University - Engineering Sciences, vol. 30, no. 4, pp. $345-354,2018$.

[7] D. J. Ramos and G. B. Daniel, "Evaluation of bearing's cavitation effects on the rotor dynamic behavior," Applied Mathematical Modelling, vol. 77, pp. 49-65, 2020.

[8] S. Aggarwal and R. K. Pandey, "Frictional and load-carrying behaviors of micro-textured sector shape pad thrust bearing incorporating the cavitation and thermal effects," Lubrication Science, vol. 29, no. 4, pp. 255-277, 2017.
[9] F. Cheng and W. X. Ji, "A velocity-slip model for analysis of the fluid film in the cavitation region of a journal bearing," Tribology International, vol. 97, pp. 163-172, 2016.

[10] G. Y. Gao, Z. W. Yin, D. Jiang, X. L. Zhang, and Y. Z. Wang, "Analysis on design parameters of water-lubricated journal bearings under hydrodynamic lubrication," Proceedings of the Institution of Mechanical Engineers, Part J: Journal of Engineering Tribology, vol. 23, no. 8, pp. 1019-1029, 2016.

[11] X. Li, S. Y. Song, and Z. R. Hao, C. W. Gu, "Cavitation mechanism of oil-film bearing and development of a new gaseous cavitation model based on air solubility," Journal of Tribology, vol. 134, no. 3, pp. 1-7, 2012.

[12] R. Guo, G. Y. Zhao, Z. F. Wang, X. J. Fu, J. G. Yang, and X. D. $\mathrm{Zhu}$, "Studies on influence of noncondensable gas in lubricating oil on performance of hydrodynamic journal bearing," Journal of Mechanical Engineering, vol. 52, no. 12, pp. 152-157, 2016.

[13] C. K. Christiansen, J. H. Walther, P. Klit, and A. Vølund, "Investigation of journal orbit and flow pattern in a dynamically loaded journal bearing," Tribology International, vol. 114, pp. 450-457, 2017.

[14] B. Ji, X. W. Luo, R. E. A. Arndt, X. Peng, and Y. Wu, "Large eddy simulation and theoretical investigations of the transient cavitating vortical flow structure around a NACA66 hydrofoil," International Journal of Multiphase Flow, vol. 68, pp. 121-134, 2015.

[15] H. Y. Cheng, X. R. Bai, X. P. Long, B. Ji, X. X. Peng, and M. Farhat, "Large eddy simulation of the tip-leakage cavitating flow with an insight on how cavitation influences vorticity and turbulence," Applied Mathematical Modelling, vol. 77, pp. 788-809, 2020.

[16] A. P. Zhang, M. N. Xie, and S. Q. Lin, "Analysis on two phase flow of oil film characteristics of elliptical bearing," Lubrication Engineering, vol. 3, pp. 64-68, 2015.

[17] H. Su, X. J. Wang, and Z. M. Zhang, "History moving of cavitation for dynamically loaded journal bearing," Chinese Journal of Mechanical Engineering, vol. 42, no. 4, pp. 8-12, 2006. 